\title{
Editorial
}

\section{Peritoneal Dialysis as the Dialysis Initial Modality of Choice for Renal Replacement Therapy Initiation: Moving from "Why" to "Why Not"}

\author{
Srinivas Subramanian ${ }^{a, b} \quad$ Muhammad Masoom Javaid $^{a, b}$ \\ a Department of Medicine, Division of Nephrology, National University Hospital, and bepartment of Medicine, \\ Yong Loo Lin School of Medicine, National University of Singapore, Singapore, Singapore
}

We nephrologists have been trained to offer peritoneal dialysis $(\mathrm{PD})$ as a comparable option for renal replacement therapy (RRT) on par with hemodialysis (HD). This is understandable in the era of "expert opinions" and "customer is king" culture where a shopper can go to a grocer and choose from any of the multitude of options available for something like fat-free milk. The authors are however perturbed by the unfair treatment that PD gets despite evidence that suggests that it is comparable in outcomes [1] but superior in costs [2], convenience, preservation of residual renal function (RRF) [3] and delivering a better quality of life [4]. In our opinion, PD should be offered as the default option to the patient requiring RRT and HD considered in patients who have either contraindications to $\mathrm{PD}$ or in patients who feel that $\mathrm{PD}$ is not right for them.

End-stage renal disease affects the socioeconomically disadvantaged sections of the society disproportionately in the developed world. Dialysis costs impose a financial strain in a large proportion of these patients [5]. In addition to costs to the individual, dialysis also imposes a significant burden on the economies of countries. In different contexts where costs have been studied, PD was found to be cost effective when compared with HD [2]. For those with higher dependency and limited mobility, the

\section{KARGER}

(C) 2017 S. Karger AG, Basel

E-Mail karger@karger.com

www.karger.com/bpu costs associated with ambulance and para-transit to and back from the HD centers can be significant.

PD wins over HD in the arena of convenience to the patients. PD is done in the comfort of the patient's home and allows them a limited degree of flexibility. HD requires the patients to stick to a rigid schedule in most instances. It requires commuting to the dialysis center and back except in those who are on home HD.

Preservation of RRF should be an important goal for every nephrologist. The presence of RRF is associated with lesser mortality, lesser need for hospitalizations and can help in clearance of "middle molecules" that are not well cleared by conventional HD. PD correlates with better preservation of RRF. This may, in part, explain the mortality advantage that $\mathrm{PD}$ patients enjoy especially in the early years after initiation of PD [6].

Despite the above evidence, the prevalent practice remains that when a hospitalized patient requires timely initiation of RRT, HD is the preferred modality. This is despite the evidence that PD can be initiated in a timely and effective manner. Patients who are initiated on PD urgently tend to stay on PD long term. When HD is initiated urgently, this is done so through a tunneled or a nontunneled dialysis catheter. The use of dialysis catheter comes with additional risks of catheter-related blood 
stream infections, catheter dysfunction, catheter-associated venous thromboembolism and other complications related to catheter placements. This needs to be balanced against the somewhat lower risk of PD catheter-associated infections and the rarity of blood stream infections associated with the PD catheter. The other disadvantages of urgently initiated PD include derangements in the glucose level in susceptible patients, risks of leaks and hernias, PD catheter dysfunction, inability to titrate the ultrafiltration with precision unlike HD and slower correction of metabolic abnormalities when compared to HD. The authors have first-hand experience with "urgent start" PD with good outcomes.
One reason that practices do not change is the inertia of the doctors, hospitals and the health systems. "This is how things have been done all along" and "Why do we need to change things when things are working ok?" have been questions that we are faced when we try to implement changes for the better. In the author's experience, the urgent initiation of PD is safe and effective. We are duty bound to deliver care that is evidence based and cost effective. Yet, as nephrologists, we allow our biases to cloud our ability to give the best possible advise to the patients we serve. Changing our practices in light of the evolving evidence is necessary to provide good service to our patients.

\section{References}

1 Yang F, Khin LW, Lau T, Chua HR, Vathsala A, Lee E, Luo N: Hemodialysis versus peritoneal dialysis: a comparison of survival outcomes in South-East Asian patients with end-stage renal disease. PLoS One 2015;10: e0140195.

2 Yang F, Lau T, Luo N: Cost-effectiveness of haemodialysis and peritoneal dialysis for patients with end-stage renal disease in Singapore. Nephrology (Carlton) 2016;21:669-677.
3 Lang SM, Bergner A, Töpfer M, Schiffl H: Preservation of residual renal function in dialysis patients: effects of dialysis-techniquerelated factors. Perit Dial Int 2001;21:52-57.

4 Cameron JI, Whiteside C, Katz J, Devins GM: Differences in quality of life across renal replacement therapies: a meta-analytic comparison. Am J Kidney Dis 2000;35:629-637.
5 Crews DC, Gutiérrez OM, Fedewa SA, Luthi JC, Shoham D, Judd SE, Powe NR, McClellan WM: Low income, community poverty and risk of end stage renal disease. BMC Nephrol 2014; 15:192.

6 Kumar VA, Sidell MA, Jones JP, Vonesh EF: Survival of propensity matched incident peritoneal and hemodialysis patients in a United States health care system. Kidney Int 2014;86: 1016-1022. 